\title{
Collagenases and cracks in the plaque
}

Peter Libby

Division of Cardiovascular Medicine, Department of Medicine, Brigham and Women’s Hospital, Harvard Medical School, Boston, Massachusetts, USA

The core of an atheromatous plaque contains lipids, macrophages, and cellular debris, typically covered by a fibrous cap that separates the thrombogenic core from the blood. Rupture of the fibrous cap causes most fatal myocardial infarctions. Interstitial collagen confers tensile strength on the cap, as it does in skin and tendons. In 1994, Peter Libby and colleagues demonstrated overexpression of collagenolytic enzymes in atheromatous plaques and implicated MMPs in the destabilization of these lesions.

The formation of plaques within the arterial intima characterizes atherosclerosis. Plaques typically consist of a lipid core covered by a fibrous cap rich in extracellular matrix, produced largely by arterial smooth muscle cells (Figure 1A). Most fatal myocardial infarctions result from a fracture in the plaque's fibrous cap (1). The fibrous cap derives tensile strength from interstitial collagen, as do skin and sinew. Twenty years ago, my colleagues and I proposed the hypothesis that excessive collagen catabolism could weaken the fibrous cap and thus render plaques prone to rupture (2). Our laboratory, focused on inflammatory signaling in vascular biology and atherogenesis, tested whether inflammatory mediators could elicit overexpression of interstitial collagenases - enzymes that catalyze the first step in collagen catabolism (Figure 1B).

\section{The collagenase quest}

My colleagues and I demonstrated the induction of interstitial collagenase in human vascular smooth muscle cells by IL-1, a cytokine that we had linked previously to vascular inflammation $(3,4)$. Our 1994 article in the JCI extended these in vitro observations to human atherosclerosis, demonstrating overexpression of the interstitial collagenase MMP-1, among other MMPs, in human atherosclerotic lesions (4). These studies found that foam cells bearing markers of smooth muscle or macrophage origin overexpressed MMP-1, without raising the levels of the endogenous inhibitors (tissue inhibitors of MMPs, e.g., TIMP-1). Further

Conflict of interest: Peter Libby is a consultant for Pri-Med and Interleukin Genetics. He receives research support from Novartis and General Electric.

Citation for this article: J Clin Invest. 2013; 123(8):3201-3203. doi:10.1172/JCI67526. studies identified elevated levels of two other interstitial collagenases (MMP-8 and MMP-13) and localized a neoepitope uncovered by collagenase cleavage in situ in human plaques (Figure 1B and refs. 5, $6)$. Other groups also implicated MMPs in arterial biology $(7,8)$.

Our subsequent studies tested whether lipid loading of mononuclear phagocytes would lead to collagenase overexpression (Figure 1B). To sidestep laboratory artifice, my colleagues and I used rabbits as in vivo "incubators." Foam cells in atherosclerotic plaques of hypercholesterolemic rabbits contained abundant MMP-1, while alveolar macrophages in the same animals showed negligible MMP-1 expression (9). This ensemble of experiments presented a coherent but circumstantial set of observations that supported a role for MMP interstitial collagenase action in determining the collagen content of atherosclerotic plaques.

\section{Closing the loop of causality}

To determine directly whether MMPs regulated the collagen content of atherosclerotic plaques, we used genetic and pharmacologic approaches to induce gain or loss of collagenase function in atherosclerotic mice. Mice with a "knockin" mutation that rendered interstitial collagen resistant to MMP collagenases showed accentuated collagen accumulation in plaques (10). Conversely, mice lacking MMP-13, a principal interstitial collagenase in mice, or receiving a selective inhibitor of MMP-13 showed similar collagen accumulation (11). Ex vivo biomechanical analysis showed that collagen accumulation actually increased the tensile strength of an atherosclerotic aorta (12). All of these pieces pointed to a key role of collagen breakdown in controlling the content of this critical component of the plaque's fibrous cap - all that stands between many patients and the acute thrombotic complications of atherosclerosis.

\section{Lipid lowering calms collagenolysis}

Another avenue of research tested the hypothesis that therapeutic interventions that reduced atherosclerotic events in humans would limit collagenase expression and increase collagen content of experimental atherosclerotic plaques. Rabbit experiments showed that dietary lipid lowering reduced MMP-1 expression and increased plaque collagen content (13). Other experiments tested whether statin treatment could achieve similar alterations in collagen economy. These experiments used Watanabe heritable hyperlipidemic (WHHL) rabbits, a strain with reduced LDL receptor function. Although WHHL rabbits showed only modestly decreased serum cholesterol when treated with statins, these agents decreased lesional collagenase activity and increased collagen content (14). Lipid lowering through diet or statins decreased plaque thrombogenicity by reducing tissue factor expression and limited other manifestations of inflammatory activation $(15,16)$. This body of observations provided insight into the cellular and molecular mechanisms by which lipid-lowering therapy reduced clinical atherothrombotic events. Observations in human atherosclerotic plaques using histopathology and various imaging modalities similarly showed an increase in the estimated fibrous character of plaques from humans treated with statins $(17,18)$. Despite the limitations of these observations in humans, they support the pathobiologic principles demonstrated by our animal experiments.

\section{Unanswered questions}

Although satisfying, this body of research leaves many important questions unanswered. First, plaques contain many non-metalloproteinases, and proteolytic enzymes have functions beyond matrix breakdown. Cysteinyl proteinases, for example, also alter arterial biology during atherogenesis (19). MMPs process non-matrix 
A

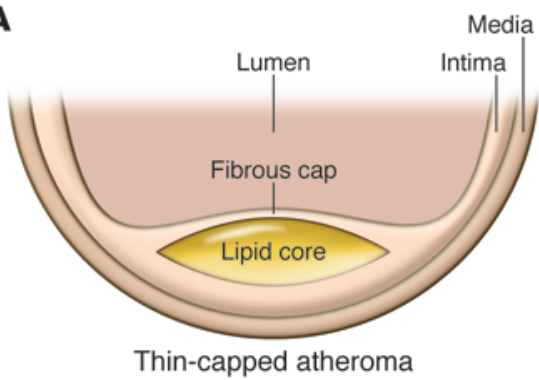

B

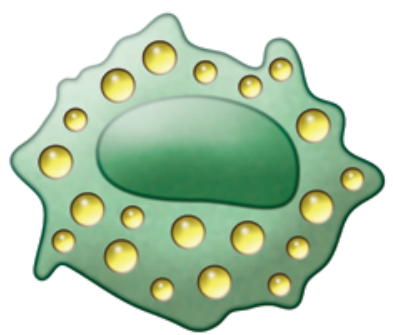

Lipid-laden macrophage

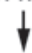

Procollagenases, MMP-1/8/13

TIMPs $-\mid \begin{aligned} & \text { ROS } \\ & \text { Plasmin, thrombin, etc. }\end{aligned}$

Active collagenases

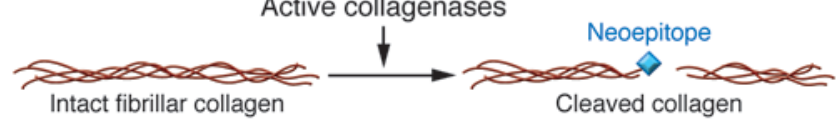

substrates - among them, cytokines and chemokines - sometimes activating them and other times inactivating them (20). Unraveling the roles for different classes of these enzymes in various aspects of arterial remodeling will require further experiments.

My group's observations in 1994 fueled the trend of using "vulnerable plaques" or "thin-capped fibroatheroma" - terms that have gained daily clinical currency as well as the concept that inflammation begets proteolysis that renders plaques "vulnerable." Yet, these simple schemata don't suffice to explain the clinical biology of plaque disruption. Most proteinase-rich plaques probably evade rupture. The number of such plaques by far exceeds clinical events, challenging the "vulnerable plaque" concept. Even when inflamed and collagenase-packed plaques do rupture, the vast majority of these disruption events pass unnoticed. Instead, cycles of subclinical disruption and healing may promote the progression of plaques from lipid-rich atheromatous lesions to those with more fibrous and calcified character that may cause flow-limiting stenosis but have less tendency to rupture.

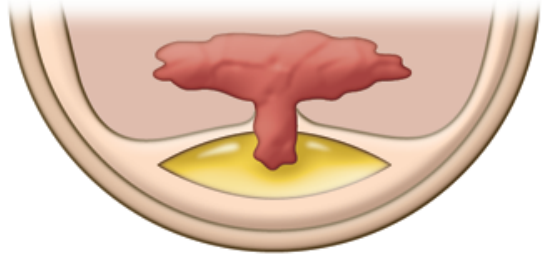

Ruptured plaque with thrombus

\section{Figure 1}

Regulation of collagen metabolism and the thrombotic complications of atherosclerosis. (A) A cross section of an artery containing a thin-capped atheroma with low collagen content due to decreased synthesis and increased breakdown of collagen is shown. A thin and weakened fibrous cap overlies a lipid-rich core in the center of the plaque (left). A plaque in which the fibrous cap has ruptured, exposing the blood coagulation components to thrombogenic material in the lipid core, triggering thrombosis, is also shown (right). (B) Lipid-laden macrophages in atherosclerotic plaques express MMPs. Various extracellular stimuli, including reactive oxygen species, plasmin, and thrombin, contribute to enzymatic activation of these enzymes that is required for their proteolytic activity. Macrophages in human plaques overexpress the three known MMP interstitial collagenases, MMP-1, MMP-8, and MMP13. The endogenous inhibitors of this family of enzymes, the tissue inhibitors of MMPs (TIMPs), do not change markedly between unaffected and atherosclerotic arteries. The MMP interstitial collagenases catalyze the initial proteolytic cleavage of intact triple helical fibrillar collagen.
So-called "vulnerable plaques" can persist for decades, yet an acute event can develop in an instant. What determines when a particular plaque will rupture and provoke a clinical event? Plaque disruption requires not only a suitable anatomic substrate, but also a triggering event. Candidate triggers include catecholamines, vasospasm, and increased circumferential strain. The consequence of a given plaque disruption also depends on the "fluid phase" of blood (21). High levels of fibrinogen and plasminogen activator inhibitor-1 in plasma promote persistence and propagation of thrombus. The susceptibility of a plaque to rupture likely reflects the systemic milieu. For example, after acute tissue injury, such as that caused by a myocardial infarction or stroke, a systemic inflammatory response can enhance the inflammatory activation and protease activity in atherosclerotic plaques, potentially increasing their propensity to rupture and provoke thrombosis (22). Additionally, the biomechanical lability of the fibrous cap likely depends on more than its collagen content. Recent computational studies indicate that microcalcification can lead to inhomogeneities of material properties that greatly enhance the propensity of a plaque to rupture under strain (23). Clinical observations associating spotty calcification within plaques with clinical events support these computational exercises (24).

Rupture of the fibrous cap does not account for all fatal coronary artery thrombi in humans. Superficial erosion of the intima without frank plaque rupture accounts for more than one-fifth of fatal coronary thromboses (25). Given the effects of statins and lipid lowering on the structure of plaques described above, in the current era, rupture of the fibrous cap as a mechanism of coronary thrombosis might wane as superficial erosion increases. The proportion of acute coronary syndromes due to ST-segment elevation myocardial infarction (STEMI) has dropped, while the contribution of non-ST segment elevation myocardial infarction (NSTEMI) has risen (26). Could statins have altered the biology of acute coronary syndromes such that plaque rupture has become less important as a cause?

In scientific stories, as in good novels, as one chapter closes, the narrative often raises unresolved conflicts to explore in coming pages. In medicine, we never reach the final 
chapter - the denouement seems eternally beyond reach. We must relentlessly strive to solve the next layer of complexity uncovered in our Sisyphean quest to understand nature and to advance our art.

\section{Acknowledgments}

Peter Libby is supported by a grant from the National Heart, Lung, and Blood Institute (HL-80472).

Address correspondence to: Peter Libby, Division of Cardiovascular Medicine, Department of Medicine, Brigham and Women's Hospital, Harvard Medical School, 77 Avenue Louis Pasteur, Boston, Massachusetts 02115, USA. Phone: 617.525.4383; Fax: 617.525.4999; E-mail: plibby@partners.org.

1. Falk E, Nakano M, Benton JF, Finn AV, Virmani R. Update on acute coronary syndromes: the pathologists' view. Eur Heart J. 2013;34(10):719-728.

2. Libby P, Clinton SK. The role of macrophages in atherogenesis. Curr Opin Lipidol. 1993;4(5):355-363.

3. Galis Z, et al. Cytokine-stimulated human vascular smooth muscle cells synthesize a complement of enzymes required for extracellular matrix digestion. Circ Res. 1994;75(1):181-189.

4. Galis Z, Sukhova G, Lark M, Libby P. Increased expression of matrix metalloproteinases and matrix degrading activity in vulnerable regions of human atherosclerotic plaques. $J$ Clin Invest. 1994;94(6):2493-2503.

5 . Sukhova GK, et al. Evidence for increased collage- nolysis by interstitial collagenases- 1 and -3 in vulnerable human atheromatous plaques. Circulation. 1999;99(19):2503-2509.

6. Herman MP, et al. Expression of neutrophil collagenase (matrix metalloproteinase-8) in human atheroma: a novel collagenolytic pathway suggested by transcriptional profiling. Circulation. 2001;104(16):1899-1904.

7. Henney AM, et al. Localization of stromelysin gene expression in atherosclerotic plaques by in situ hybridization. Proc Natl Acad Sci U S A. 1991;88(18):8154-8158.

8. Nikkari ST, et al. Interstitial collagenase (MMP-1) expression in human carotid atherosclerosis. Circulation. 1995;92(6):1393-1398.

9. Galis Z, Sukhova G, Kranzhöfer R, Clark S, Libby P. Macrophage foam cells from experimental atheroma constitutively produce matrix-degrading proteinases. Proc Natl Acad Sci U S A. 1995;92(2):402-406.

10. Fukumoto Y, et al. Genetically determined resistance to collagenase action augments interstitial collagen accumulation in atherosclerotic plaques. Circulation. 2004;110(14):1953-1959.

11. Deguchi JO, et al. Matrix metalloproteinase-13/ collagenase-3 deletion promotes collagen accumulation and organization in mouse atherosclerotic plaques. Circulation. 2005;112(17):2708-2715.

12. Deguchi J, et al. Genetically engineered collagenase resistance promotes abdominal aortic aneurysm formation. Lab Invest. 2009;89(3):315-326.

13. Aikawa $M$, et al. Lipid lowering by diet reduces matrix metalloproteinase activity and increases collagen content of rabbit atheroma: a potential mechanism of lesion stabilization. Circulation. 1998;97(24):2433-2444.

14. Fukumoto $Y$, et al. Statins alter smooth muscle cell accumulation and collagen content in established atheroma of watanabe heritable hyperlipidemic rabbits. Circulation. 2001;103(7):993-999.

15. Aikawa M, et al. Dietary lipid lowering reduces tissue factor expression in rabbit atheroma. Circulation. 1999;100(11):1215-1222.
16. Aikawa M, et al. An HMG-CoA reductase inhibitor, cerivastatin, suppresses growth of macrophages expressing matrix metalloproteinases and tissue factor in vivo and in vitro. Circulation. 2001;103(2):276-283.

17. Crisby M, Nordin-Fredriksson G, Shah PK, Yano J, Zhu J, Nilsson J. Pravastatin treatment increases collagen content and decreases lipid content, inflammation, metalloproteinases, and cell death in human carotid plaques: implications for plaque stabilization. Circulation. 2001;103(7):926-933.

18. Watanabe K, et al. Stabilization of carotid atheroma assessed by quantitative ultrasound analysis in nonhypercholesterolemic patients with coronary artery disease. J Am Coll Cardiol. 2005;46(11):2022-2030.

19. Liu J, Sukhova GK, Sun JS, Xu WH, Libby P, Shi GP. Lysosomal cysteine proteases in atherosclerosis. Arterioscler Thromb Vasc Biol. 2004;24(8):1359-1366.

20. Schoenbeck U, Mach F, Libby P. Generation of biologically active IL-1 beta by matrix metalloproteinases: a novel caspase-1-independent pathway of IL-1 beta processing. I Immunol. 1998;161(7):3340-3346.

21. Libby P, Theroux P. Pathophysiology of coronary artery disease. Circulation. 2005;111(25):3481-3488.

22. Dutta P, et al. Myocardial infarction accelerates atherosclerosis. Nature. 2012;487(7407):325-329.

23. Maldonado $\mathrm{N}$, et al. A mechanistic analysis of the role of microcalcifications in atherosclerotic plaque stability: potential implications for plaque rupture. Am J Physiol Heart Circ Physiol. 2012;303(5):H619-H628.

24. Ehara S, et al. Spotty calcification typifies the culprit plaque in patients with acute myocardial infarction: an intravascular ultrasound study. Circulation. 2004;110(22):3424-3429.

25. Libby P. Mechanisms of the acute coronary syndromes and their implications for therapy. $N$ Engl J Med. 2013;368(21):2004-2013.

26. Yeh RW, Sidney S, Chandra M, Sorel M, Selby JV, Go AS. Population trends in the incidence and outcomes of acute myocardial infarction. NEnglJ Med. 2010;362(23):2155-2165. 